\title{
High Availability Green Gear-shifting Mechanism in Cloud Storage System
}

\author{
Li Zhou $^{1,2}$, Chi Dong ${ }^{1,2}$, Xindong You ${ }^{* 1,2}$, Jie Huang ${ }^{1,2}$ and Congfeng Jiang ${ }^{1,2}$ \\ ${ }^{1}$ School of Computer Science, Hangzhou Dianzi University, Hangzhou, \\ China \\ ${ }^{2}$ Key Laboratories of Complex Systems Modeling and Simulation, Ministry of \\ Education \\ *Corresponding author: youxindong@hdu.edu.cn
}

\begin{abstract}
The energy problem is one of the serious problems in the current large-scale storage systems need to be addressed urgently. In order to reduce the energy consumption of cloud storage system, and to meet the performance needs of users, this paper purposed a cloud storage system integrated high availability green gear-shifting mechanism (HGLG): The frame designed a new data partitioning strategy, data replication management strategy and proposed energy gear-shifting mechanism based on the data partition and data replication management. Data partitioning strategy divide data into cold data, hot data, seasonal data and the new data and place it in the appropriate zone through green data classification strategy based on anticipation (AGDC). Depending on the nature of the data, the paper accordingly designed replica placement and replica number. Based on the above data partitioning, data replication management, this paper presents energy gear-shifting mechanism, which automatically gear-shifting through neural network model predicting follow-up period assignments. Experiments based on Grid Sim show that: the energy consumption of gear-shifting mechanisms cost effective, which saved about $43 \%$ average energy at the expense of about 1.6ms average response time and the maximum energy savings is about $70 \%$.
\end{abstract}

Keywords: data partitioning, data replication management, gear-shifting mechanism

\section{Introduction}

With the increasing popularity of data-intensive applications and services, large-scale data centers consumed enormous power resource. The energy consumption of storage system accounting for 25 to $35 \%$ [8] of data centers energy consumption. With various application requiring storage devices of a $60 \%$ annual growth rate, the energy consumed of storage system will not be ignored. The cloud storage has become a trend in the future development of storage; Cloud Storage System has been widely used. Therefore, how to reduce cloud storage devices energy consumption in large data center is an urgent problem need to be solved.

A lot of research has been done on cloud storage consumption, part of which is based on data classification, data replication, data placement, data gear-shifting mechanism. According to an article in which the FREP above strategy theoretically with minimal performance cost in exchange for a 90 percent energy savings, but the presence of some of the problems FERP model source data backup in the number of non-CS node may lead to excessive, considering only the overall increase in base node and did not consider the case when a node storing data reaches saturation, the new node's situation and other issues. To solve these problems, we propose HGLG framework: The framework proposes new data partitioning strategy, data replication management and energy gear-shifting mechanism, and address how the different nature of the data to be backed up, placed; 
when new data arrives on how to back up and place; how to open a new node to place the data; how to achieve the saving effect gear-shifting mechanism to meet the performance requirements and so on. Experiment results show that the HGLG system model can ensure the system performance requirements under the premise of the greatest degree of reducing energy consumption.

\section{Related Work}

A lot of research has been done on cloud storage consumption, part of which is based on data classification, data backup, data placement, data files, data lifting and other policies. Li, Hongyan proposed REST architecture [5]: by slightly changing the data layout strategies, most of the time REST can safely keep a lot of redundant storage node in the standby mode, even in case of power failure can ensure redundant nodes safety. It deploys advanced real-time workload monitoring, which provides flexible power standby or power down nodes to adapt to changes in load. Saiqin Long, Yuelong Zhao et al proposed TPES saving strategies [6]: they are based on variable factors backup management strategy, based on the best total cost of reconfiguring the cluster state transition strategy and tactics based on workload prediction and observation. Liu Jingyu .et proposed S-RAID structure which uses SSD disks mixed with ordinary [2] disk, by closing part of theidle disk to save energy. Experiments show that the hybrid S-RAID5 disk which composed of 12 general disks and two solid-state SSD disk compared with the same level RAID5, energy consumption of hybrid S-RAID5 disk is only $28 \%$.AutoMig [3] Comprehensive parameters such as history file access, file size, equipment utilization .etc., dynamic classification of documents and using the LRU queue maintains files state which in flash memory device. In hierarchical storage systems show that compared with existing methods, AutoMig effectively shortening the foreground I / O response time. Kim and Rotem were inspired on the RAID implementation PARAID mechanism proposed by Weddle proposed FREP [4] (Fractional Replication for Energy Proportionality) mechanisms: the granularity of the data will be backed up to the storage node extension to the system load lighter when you can shut down the entire node to achieve a greater degree of conservation land. On Disksim simulation experiments show that: compared to PARAID mechanism, FREP mechanism under the premise of more energy-efficient, has a faster response time, while a large number of simulation experiments show that the FREP mechanism could in theory to sacrifice performance in exchange for minimal $90 \%$ of energy savings.

All of the above related work show through data classification, data replication, data placement, data gear-shifting mechanism. According to an article in which the FREP above strategy theoretically with minimal performance cost in exchange for a 90 percent energy savings, but the presence of some of the problems FERP model source data backup in the number of non-CS node may lead to excessive, considering only the overall increase in base node and did not consider the case when a node storing data reaches saturation, the new node's situation and other issues. To solve these problems, we propose HGLG framework: The framework proposes new data partitioning strategy, data replication management and energy gear-shifting mechanism, and address how the different nature of the data to be backed up, placed; when new data arrives on how to back up and place; how to open a new node to place the data; how to achieve the saving effect gear-shifting mechanism to meet the performance requirements and so on. Data partitioning strategy through green data classification strategy based on anticipation (AGDC) divides data into the cold data, hot data, seasonal data and the new data and put it into the appropriate zone. Depending on the nature of the data, this paper develops the appropriate number of replica and replica placement. Based on the above data partitioning, data replication management, this paper proposed energy gear-shifting mechanism that automatically gear-shifting through neural network model to predict follow-up period 
assignments. By HGLG system model can guarantee the system performance requirements under the premise of the greatest degree of reducing energy consumption. Experiment results show that the HGLG system model can ensure the system performance requirements under the premise of the greatest degree of reducing energy consumption.

\section{HGLG System Model}

HGLG system model is combined with dynamic voltage management technology and based on anticipation of the green data classification strategy. Green data classification strategy based on anticipation (AGDC), which classifies the data in cloud storage system: the hot data is stored in the hot disk zone; the cold data is stored in the cold disk zone. AGDC employ neural-network prediction on seasonal data, predicting the temperature of data in the next period, executing seasonal data migration in cold and hot regions. AGDC also adopt a new correlating algorithm on new data, analyzing its relations with old data in the storage system and predicting the data temperature. HGLG system model proposed new data partitioning strategy, data replication management strategy, based on the basic data partition and data replication proposed gear-shifting mechanism.

In this paper, the data will be divided into five category, they are new data vold data hot data v cold data、 seasonal data. The following is the definition of these different kinds of data.

Data Temperature: The higher average number of data access operation is, the higher data temperature is, on the contrary, the lower data temperature is. Factors that directly affect the data temperature are the times of data to be operated.

New data: data which does not exist or less time in cloud storage system, and times that data be operated is zero or less.

Old data: data that exists a period of time in cloud storage system.

Cold data: old data that the average number of data access operation is less than cold temperature threshold.

Hot data: old data that the average number of data access operation is greater than cold temperature threshold.

Seasonal data: old data the averages of data access operation is greater than hot threshold some times, data temperature is fluctuating.

The following is the article several important definitions.

Node zone is divided into: the cold zone and hot zone;

Hot zone is divided into: source hot zone, the first backup zone, the second backup zone, the 3 rd zone ... the k-th backup zone (size $\mathrm{k}$ is determined by the number of nodes, under normal circumstances $\mathrm{k}<=4$ );

Cold zone is divided into: source cold zone, backup zone (cold data contains only one backup);

The i-th backup zone: the first storage zone of the i-th backup data zone of the hot source of the data, not all data of the i-th backup are present, only a certain percentage of a certain property of the existence of the data was backed up. The larger the value of $i$, the less there is the backup data;

Source data zone: source hotzone, source cold zone;

Node status are divided into:dormant status and active status, and active status is divided into positive status and ideal status;

Dormant status: the node is dormant, not to accept the task request in low power status;

Active status: that node can work, accept task request and process tasks;

Positive status: the node status is processing tasks;

Ideal status: the node is active but no processing tasks, in an idle status. 


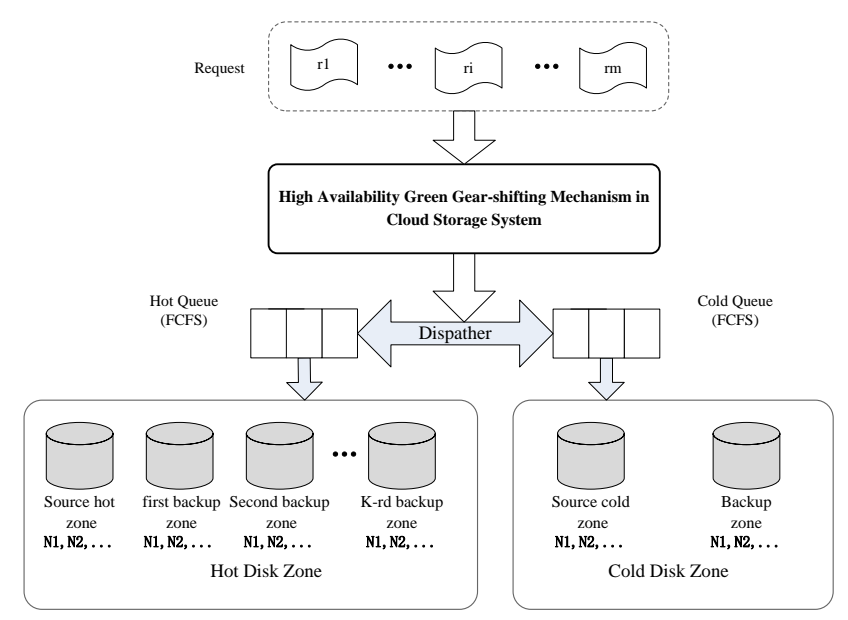

Figure 1. HGLG Chart

HGLG automatically gear-shifting system model based on the current load on the system, when the system load is heavy upshift, when the system load is light downshift. Figure 1 depicts the architecture diagram of HGLG system, when the system load is light, it can only open source hot zone and source cold zone; when systemload graduallyincrease, it can gradually opened some nodes of first backup zone; when the first backup zone fully open or cannot meet the current load, backup zone gradually open the node of second backup zone, and so on. When the loadgradually reduce, HGLG can be gradually closed the node of maximum backup zone, and gradually close the backup zone. Gear-shifting unit ofHGLG system model may be based on a node or azone.

Table 1. Parameter Description

\begin{tabular}{|c|c|}
\hline Parameter Description & Parameter Description \\
\hline $\mathrm{HSN}_{j}$ & j-th node of the source hot zone \\
\hline $\mathrm{CSN}_{j}$ & j-th node of the source cold zone \\
\hline $\mathrm{HN}_{i j}$ & j-th node of the i-rd backup zoneof hot zone \\
\hline $\mathrm{CN}_{j}$ & $\mathrm{j}$-th node in backup zone of cold zone \\
\hline hsn & $\begin{array}{l}\text { The number of source hot block insource hot zone (Note: } \\
\text { In the hsn-th node there may also be the source hot } \\
\text { blockand the source seasonal hot block) }\end{array}$ \\
\hline $\operatorname{csn}$ & $\begin{array}{l}\text { The number of source cold block in source cold zone } \\
\text { (Note: In the csn-th node there may also be the source } \\
\text { cold blockand the source seasonal cold block) }\end{array}$ \\
\hline hssn +1 & $\begin{array}{l}\text { The number of source seasonal hot block in source hot } \\
\text { zone }\end{array}$ \\
\hline $\operatorname{cssn}+1$ & $\begin{array}{l}\text { The number of source seasonal cold block in source cold } \\
\text { zone }\end{array}$ \\
\hline$\left|\mathrm{hsn} * a_{j} \%\right|$ & $\begin{array}{l}\text { the number of nodes required for storage } a_{j} \% \text { data of } \\
\text { hsnsource hot blocks }\end{array}$ \\
\hline
\end{tabular}

\subsection{Data Partition Strategy}

In this section, we through green data classification strategy based on anticipation (AGDC) divide data into the cold data, hot data, seasonal data and the new data and put it into the appropriate zone.

Data partition steps:

1) The initial data divided by green data classification strategy based on anticipation 
(AGDC), and mark it, turn 2);

2) The cold data putted into the sourcecold zone, the sourcecold zone contains only one nodeat the initial moment, only when the node current is full (full definition of a node refers to a saturated status, can independently define), HGLG opening a new node as a node of the source coldzone storage data, turn 3)

3) The new data and seasonal data which pre-divided for cold individually placed in source coldzone of cold zone turn 4);

4) The hot data putted into the source hot zone, the source hot zone contains only one node at the initial moment, only when the node current is full (full definition of a node refers to a saturated status, it also can independently define), HGLG opening a new node as a node of the source hot zone storage data, number of nodes from low to high, thetemperature of data gradually reduce, turn 5);

5) The new data and seasonal data which pre-divided for hot individually placed in opened node of source hot zone, if and only if the current node filled, before opening a new node to store data. At the node of convergence may exist source hot data and source seasonal hot data. Starting number of seasonal data storage greater than or equal last node number of stored hot data.

\subsection{Data Replication Management}

Good data replication management determines the security and availability of data, data replication management also determines the feasibility of gear-shifting mechanism, and therefore essential to develop a viable data replication management. Depending on the nature of the data, this chapter develops the appropriate number of replica and replica placement. At the same time this chapter also solve issuesthat when a new write request arrives, where the new data placed and how to replication.

3.2.1. Determining the ReplicaNumber and Placement : In this section, we designed a data replication management. The node zone is divided into cold zone and hot zone, hot zone containing the source hotzone, the first backup zone, the second backup zone ... m-rd backup zone; cold zone contains source cold zone and backup zone. We assume that after the thermal partition processing 3.1, the node $\mathrm{HSN}_{j}$ of source hot zone contained source hotblockHSD ${ }_{j}$, the source seasonalhot block is $\mathrm{HSSD}_{j}$, the node $\mathrm{CSN}_{j}$ of source cold zone contained source cold block $\operatorname{CSD}_{j}$, and thesource seasonalcold block is $\operatorname{CSSD}_{j}$. The number of backup zone in hot zone is $\mathrm{m}$, the hot data ratio in i-th backup zone of hot zoneis $a_{i} \%$. We assume that source hot block in i-rd backup zone of hot zone is $\left\{\mathrm{HSD}_{1}, \mathrm{HSD}_{2}, \ldots \mathrm{HSD}_{h s n}\right\}\left(a_{i} \%\right)$, of which only the 1,2 backup zonecontaining source seasonal hotblock $\left\{\operatorname{HSSD}_{h s n}, \mathrm{HSSD}_{h s n+1}, \ldots \mathrm{HSSD}_{h s n+h s s n}\right\}$, no other backup zone containing this data blocks, namely seasonal data only has 2 backup and $a_{1} \%, a_{2} \%$ default value is generally $100 \%$. All data in the cold zone contains only one backup.

The value of $\mathrm{m}$ and $a_{i} \%$ determination (by default even if the node capacity is not sufficient, but the hot can reach the 2 data backup)

1) The data in source hotzone andsource cold zone $100 \%$ backup, this backup zoneof source hotzone called the first backup zone, this backup zone of source cold zone called the backup zone, the remaining nodes is $\mathrm{s} 1=\mathrm{n}-\mathrm{hsn}-$ hssn - csn - cssn, turn 2);

2) If the data $s 1>0 \& \& s 1 \geq h s n+h s s n$, then data in source hotzone $100 \%$ full backup, this backup zone called a second backup zone, the remaining nodes $\mathrm{s} 2=\mathrm{s} 1-\mathrm{hsn}-\mathrm{hssn}$, turn 3 );

3) If $\mathrm{s} 2>0$ and $\mathrm{s} 2 \geq\left|\mathrm{hsn} * a_{3} \%\right|$, then data before ranking $a_{3} \%$ of source hot zone is backed up, the remaining nodes iss $3=\mathrm{s} 2-\left|\mathrm{hsn} * a_{3} \%\right|$, 
thiszone known as3 backup zone, turn 4). Otherwise, the source hot data by ranking each backup to the new node until the remaining nodes filled, thiszone known as 3 backup zone, back end;

4) If $\mathrm{s} 3>0$ and $\mathrm{s} 3 \geq\left|\mathrm{hsn} * a_{4} \%\right|$, then data before ranking $a_{4} \%$ of source hot zone is backed up, the remaining nodes iss $4=\mathrm{s} 3-\left|\mathrm{hsn} * a_{4} \%\right|$, this zone known as4 backup zone, turn 4). Otherwise, the source hot data by ranking each backup to the new node until the remaining nodes filled, this zone known as 4 backup zone, back end;

5) If $\mathrm{s} 4>0$ and $\mathrm{s} 4 \geq\left|\mathrm{hsn} * a_{5} \%\right|$, then data before ranking $a_{5} \%$ of source hot zone is backed up, the remaining nodes iss $5=\mathrm{s} 4-\left|\mathrm{hsn} * a_{5} \%\right|$, this zone known as 5 backup zone, turn 4). Otherwise, the source hot data by ranking each backup to the new node until the remaining nodes filled, this zone known as 5 backup zone, back end;

The above algorithm node filled the concepts in this article assume disk capacity utilization reached $90 \%$, for some unstable disk, disk usage can be appropriately reduced. This article relates to the maximum number of backup is 5, due to individual differences in the system, the maximum number of backups can also be appropriate to increase, and increases the maximum number of backups and reduce data placement does not affect the entire layout.

Table 2. Data Backup Strategy to Achieve In Nine Nodes System

\begin{tabular}{|c|c|c|c|c|c|c|c|c|c|}
\hline & \multicolumn{5}{|c|}{ Hot zone } & \multicolumn{4}{|c|}{ Cold zone } \\
\hline zone & $\begin{array}{c}\text { Source } \\
\text { hot } \\
\text { zone }\end{array}$ & $\begin{array}{c}\text { First } \\
\text { backu } \\
\text { p } \\
\text { zone }\end{array}$ & $\begin{array}{c}\text { Second } \\
\text { backup } \\
\text { zone }\end{array}$ & $\begin{array}{c}\text { Third } \\
\text { backup } \\
\text { zone }\end{array}$ & $\begin{array}{c}\text { Fourth } \\
\text { backup } \\
\text { zone }\end{array}$ & $\begin{array}{r}\text { Sourc } \\
\mathrm{zC}\end{array}$ & $\begin{array}{l}\text { e cold } \\
\text { ne }\end{array}$ & Backu & zone \\
\hline node & $\mathrm{HSN}_{1}$ & $\mathrm{HN}_{11}$ & $\mathrm{HN}_{21}$ & $\mathrm{HN}_{31}$ & $\mathrm{HN}_{41}$ & $\mathrm{CSN}_{1}$ & $\mathrm{CSN}_{2}$ & $\mathrm{CN}_{1}$ & $\mathrm{CN}_{2}$ \\
\hline $\begin{array}{c}\text { Source } \\
\text { data }\end{array}$ & $\begin{array}{r}\mathrm{HSD}_{1} \\
\mathrm{HSSD}_{1}\end{array}$ & & & & & $\mathrm{CSD}_{1}$ & $\begin{array}{l}\mathrm{CSD}_{2} \\
\mathrm{CSSD}_{2}\end{array}$ & & \\
\hline backup & & $\begin{array}{l}\mathrm{HSD}_{1} \\
\mathrm{HSSD}_{1}\end{array}$ & $\begin{array}{c}\mathrm{HSD}_{1} \\
\mathrm{HSSD}_{1}\end{array}$ & $\begin{array}{c}\mathrm{HSD}_{1} \\
\left(a_{3} \%\right) \\
\end{array}$ & $\mathrm{HSD}_{1}\left(a_{4} 0\right.$ & & & $\mathrm{CSD}_{1}$ & $\begin{array}{l}\mathrm{CSD}_{2} \\
\mathrm{CSSD}_{2}\end{array}$ \\
\hline
\end{tabular}

Table 2 shows the data replication management strategy to achieve in nine nodes system, in which the four nodeis the node in cold zone, 5 node is the node in hot zone, in the cold zone contains the source cold data and the source seasonal colddata and its backup, in the hot zone contains the source hot data and the source seasonal hotdata and its backup. The first and second backup zone containing the backup of source hotdata andsource seasonal hotdata, and the third backup zone contains only $\mathrm{a}_{3} \%$ of source hotdata, the fourth backup zone contains only $\mathrm{a}_{4} \%$ of source hotdata.

3.2.3. New Data Arrives: In this section we solve the problem about when new data arrives on how to backup and placed, how to open a new node to place the data.

When new data arrives, to deposit in the disk, we first mark on the new data, and then use green data classification strategy based on anticipation(AGDC) for new data to predict it's temperature, if the data predicted the hot, this data is stored into the not full node of source hot zone; if the node of source hotzone is full, you have to open a new node as a node of the source hot zone to store data, then new data for backup, the backup putted into the node of first backup zone and the second backup zone; if the data predicted 
the cold,this data is stored into source coldzone and backed up.

\subsection{Gear-Shifting Mechanism}

In this section, we will elaborate on the proposed Energy Gear-shifting mechanism. The mechanism is based on data partitioning strategy and data replication management strategy, and predicts the number of subsequent period's assignments through neural network model to comply the automatic gear-shifting.

This section makes the period indicate the interval of a short period of time. Assume the request arrival rate of the $\mathrm{K}$ period before the current one respectively as $\left\{v_{1}, v_{2}, \ldots, v_{K}\right\}$, where the $v_{i}$ represents the request arrival rate of the i-th period before the current one. The request arrival rate of current period is $v_{0}=\frac{n u m_{v}}{t-t_{s}}$, where the num $_{v}$ represents the total request arrival amount during the beginning of the period $t_{s}$ to the current moment $t$. According to $\left\{v_{0}, v_{1}, v_{2}, \ldots, v_{K}\right\}$, predict the request arrival rate $\mathrm{V}$ of the next period through the neural network. Assume the open notes number of current nodes as num $_{o}$, then the request arrival rate of single-node is $\mathrm{a}=\frac{v}{\text { numo }_{o}}$, the node-task processing rate is cnum, so that the arrival time of the i-th task is:

The beginning time of the $\mathrm{i}$-th task is:

$$
R T_{i}=\frac{i-1}{a}
$$

$$
P t_{i}=\frac{i-1}{\text { cnum }}
$$

Then the waiting time for the i-th task is:

$$
\mathrm{W} t_{i}=\frac{i-1}{\text { cnum }}-\frac{i-1}{a}
$$

Then the maximum waiting time for all tasks is:

$$
\mathrm{MWT}=\max _{1 \leq \mathrm{i} \leq \mathrm{a}} \mathrm{W} t_{i}
$$

Assume the maximum waiting time among all requests in the current period as Mwt, make $\mathrm{th}_{w t}$ as the waiting time threshold. When the maximum waiting time is MWT $\geq \mathrm{th}_{w t}$, the current gear will upshift. When MWT $<\mathrm{th}_{w t}$ and Mwt $<0.5 \mathrm{th}_{w t}$, it will downshift. Otherwise, it will not change. Figure 2 describes the gear-shifting architecture diagram of HGLG system, which shows a HGLG system schematic from high to low gear. High gear means that all nodes in the system are active. This gear system has the best performance but the highest energy consumption. Low gear means that only the source data area nodes in the system are active, and the backup area nodes are in a dormant state. This gear system has the lowest energy consumption.

\section{Simulations and Analysis}

This paper integrates the above HGLG algorithm into GridSim simulator, and adds the corresponding energy parameters to nodes to assess the performance of the algorithm. To verify the effectiveness of the algorithm, this paper will compare the performance and energy consumption among the non-integrating data partition algorithms cloud storage system (NPS), the integrating traditional classification algorithm cloud storage system (TDCS), and cloud storage system integrated high availability green gear-shifting mechanism (HGLG). This experiment assumes that the number of backup for NPS systems is 3 , the number of hot data backup for TDCS system is 3 , the number of cold data backup is 1, and also the new data and the seasonal hot data for TDCS system are all stored in a hot zone. The chapter compares different impact on performance and energy among three systems through different synthetic load, different proportion of new data and seasonal hot data, and different system scale to evaluate the strategy we proposed. 


\subsection{Parameter Description}

This experiment is actualized based on the cold/hot disk array simulator. The disk-related parameters are shown in Table 3.

Table 3. Disk-Related Parameters

\begin{tabular}{ll}
\hline the average positioning time of disks in the node & $5.4 \mathrm{~ms}$ \\
Storage Capacity & $128 \mathrm{~GB}$ \\
Shaft Speed & $10000 \mathrm{RPM}$ \\
high-speed disk transfer rate & $31 \mathrm{M} / \mathrm{S}$ \\
the energy consumption when high-speed disk has task & $78.08 \mathrm{~J} / \mathrm{Mb}$ \\
the energy consumption when high-speed disk has no task & $5.26 \mathrm{~J} / \mathrm{S}$ \\
low-speed disk transfer rate & $9.3 \mathrm{M} / \mathrm{S}$ \\
the energy consumption when low-speed disk has task & $55.04 \mathrm{~J} / \mathrm{Mb}$ \\
the energy consumption when low-speed disk has no task & $2.17 \mathrm{~J} / \mathrm{S}$ \\
\hline
\end{tabular}

Table 4 shows the relevant data used in the experiments and synthetic load is shown in table 5. S11, S12, S13, S21, S22, S23 is different synthetic load. This paper assumes that the request arrival distribution obeys $\exp (6), \exp (20)$, and $\exp (50)$, where $\exp (\mathrm{a})$ indicates that the request arrival distribution is subject to an exponential distribution whose average is a that the average arrival time is a ms. The default value is set to $\exp (6)$. The request arrival distribution obeys Zipf distribution. Different indices for Zipf distribution affect requests from different hot and cold files, while the index $\theta=\log \frac{A}{100} / \log \frac{B}{100}$, where A percent of all accesses were directed to B percent of files. So that we will set the value of $\theta$ as 1.5 and 1.8 , also the default value is set to 1.5 .

Table 4. Description for the Relevant Data Used In the Experiments

\begin{tabular}{ll}
\hline the total number of files & 1000 \\
file size distribution & $1 \mathrm{M}$ \\
the proportion of the new data and the seasonal hot data & $8 \%(2 \% 5 \% 8 \% 11 \% 14 \%)$ \\
simulation time & $3 * 5 * 200 \mathrm{~S}$ \\
(time zone $*$ period $*$ simulation time in each period) & \\
\hline
\end{tabular}

Table 5: Synthetic Load Table

\begin{tabular}{|c|c|c|c|c|}
\hline Trace name & Request size & $\begin{array}{c}\text { Arrival } \\
\text { distribution }\end{array}$ & time & $\begin{array}{c}\text { Arrival } \\
\text { distribution }\end{array}$ \\
\hline S11 & $1 \mathrm{M}$ & $\exp (6)$ & & $\operatorname{Zipf}(\theta=1.5)$ \\
\hline S12 & $1 \mathrm{M}$ & $\exp (20)$ & & $\operatorname{Zipf}(\theta=1.5)$ \\
\hline S13 & $1 \mathrm{M}$ & $\exp (50)$ & & $\operatorname{Zipf}(\theta=1.5)$ \\
\hline $\mathrm{S} 21$ & $1 \mathrm{M}$ & $\exp (6)$ & & $\operatorname{Zipf}(\theta=1.8)$ \\
\hline S22 & $1 \mathrm{M}$ & $\exp (20)$ & & $\operatorname{Zipf}(\theta=1.8)$ \\
\hline S23 & $1 \mathrm{M}$ & $\exp (50)$ & & $\operatorname{Zipf}(\theta=1.8)$ \\
\hline
\end{tabular}

\subsection{Impact of Different Synthetic Load on System Performance and Energy}

Experiments take different synthetic load: S11, S12, S13, S21, S22, S23, to assess HGLG system performance and energy consumption, where the default proportion of new data and seasonal hot data is $8 \%$. The results are shown in Figure 3 and Figure 4:

As shown in figure 3 and 4, the highest energy saving percentage of TDCS system is about 27 percent and the lowest is about 14\%, while the average energy saving percentage of HGLG (6:2) systems is about 59\%, and the minimum of which is about $50 \%$. The effect of HGLG system is self-evident, which saves at most about 39\% higher than the TDCS system based on the traditional data classification. As shown in Figure 4, the 
response time of HGLG system is 0.14 milliseconds higher than the TDCS system, with a maximum about $2 \mathrm{~ms}$ response time difference between the NPS system. The loss of HGLG system performance is little, while saving effect is obvious. The disparity between S11, S12 and S13 shows that the energy saving percentage is increasing with the increasing average reaching time of tasks. In summary, even in larger systems with large quantities of task request assignments HGLG can also be well positioned to meet the performance needs of users and reach nearly $50 \%$ of energy savings.

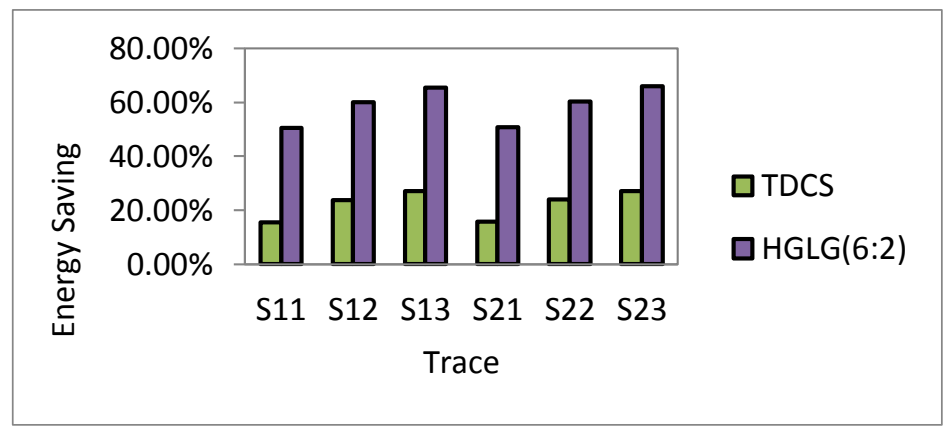

Figure 3. The Effect of Different Synthetic Load on the System Energy

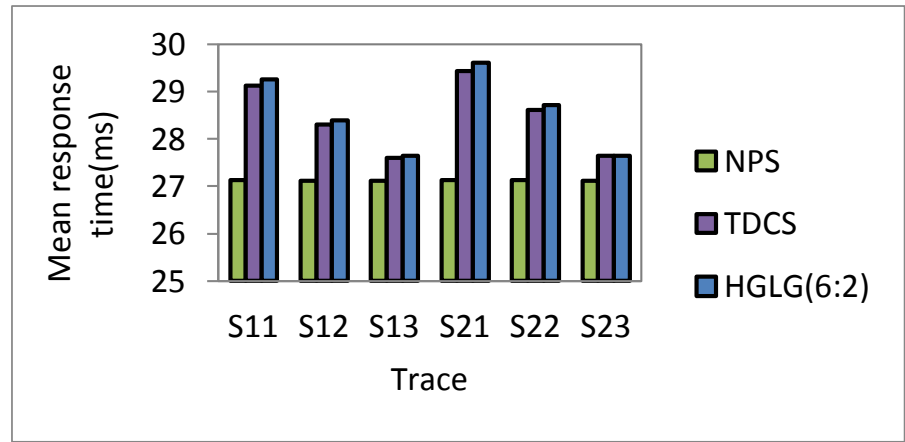

Figure 4. The Effect of Different Synthetic Load on the System Performance

\subsection{Impact of Different Proportion of New Data and Seasonal Hot Data on System Performance and Energy}

The experiments take the different proportion of new data and seasonal hot data: $2 \% 5 \%$ $8 \% 11 \% 14 \%$, to assess the HGLG system performance and the energy consumption, wherein the synthetic load is S12. The results are shown in Figure 5 and Figure 6:

As shown in Figure 5 and Figure 6, the consumption reduction percentage of TDCS, HGLG(6:2) system reduced slowly associated with increasing proportion of new data and seasonal thermal data while the consumption reduction percentage difference between the two systems gradually becomes greater. The consumption reduction percentage of TDCS system is about $22 \%$, while that of HGLG system is about $60 \%$. As shown in Figure 6 , response time of HGLG(6:2) system is about 0.4 millisecond higher than that of TDCS system, the performance differences between the systems is not obvious, but the response time of HGLG system is about 2 milliseconds higher than NPS system, the loss of system performance of HGLG is minimal. In summary, even in the new data and the seasonal hot data systems, HGLG can be very good to meet the performance needs of users and reach nearly $60 \%$ of energy savings. 


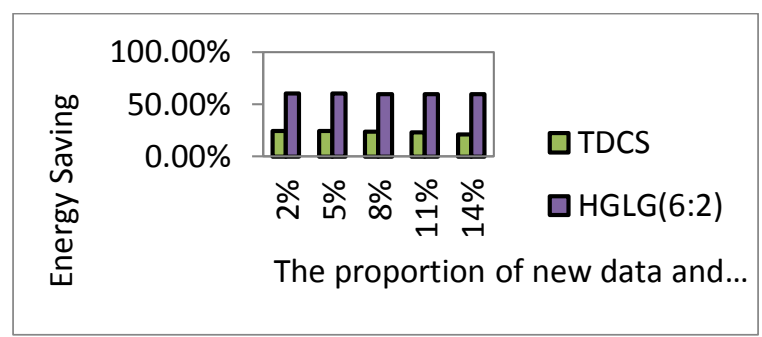

Figure 5. The Effect of the Different Proportion of New Data and Seasonal Hot Data on System Energy

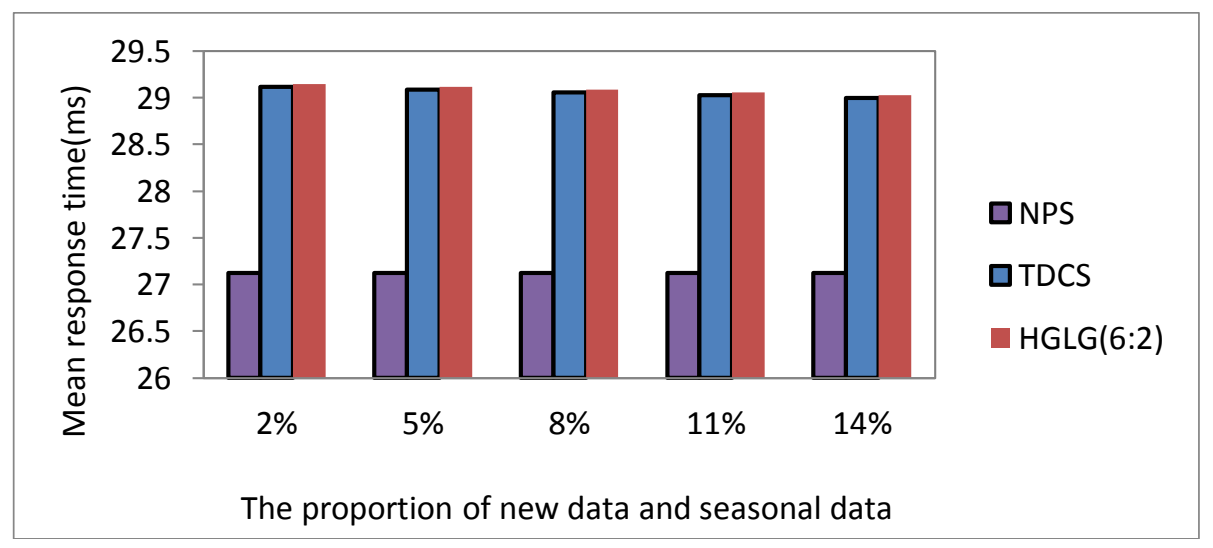

Figure 6. The Effect of the Different Proportion of New Data and Seasonal Hot Data on System Performance

\section{Conclusions and Future Work}

This paper proposes HGLG system frame: the frame design a new data partitioning strategy, data replication management strategy and proposed energy gear-shifting mechanismbased on the data partition and datareplication management. The source data zone and the backup zone of the system to distinguish in the model and the number of nodes of the zone can be increased or deleted without affecting the system architecture. Through the integrated data classification algorithm of cloud storage systems (NPS), integrated common classification algorithm of cloud storage system (TDCS) and combined with cloud storage system integrated high availability green gear-shifting mechanism (HGLG) performance and energy consumption, it is found that saving effect of HGLG systems is obvious under the premise of meeting user performance requirements, and it is generally up to about $59 \%$, the highest can reach up to $66 \%$. This system achieves a good saving effect through reasonable data partition, data replication, and gear-shifting mechanism. But there are some defects also, such as gear-shifting is up or down throughout the zone, resulting in a certain amount of wasted energy. So, next step, the main task is to minimize the additional overhead, at the same time to meet the performance requirements and make continue research to expand the policy.

\section{Acknowledgment}

This work is supported Natural Science Foundation of China (Grant No. 61402140, 61202094,6110004,61100193),by Natural Science Foundation of Zhejiang Province (LY13F0-20045, LY13F020047), Research Project of Education Office of Zhejiang Province(No. Y201222937,Y201432390), National Key Technology Research and Development Program of the Ministry of Science and Technology of China (2012BAH24B04). 


\section{References}

[1] T. Xie, SEA, A Striping-Based Energy-Aware Strategy for Data Placement in RAID-Structured Storage Systems", IEEE Transactions on Computers, vol. 6, no. 57, (2008).

[2] L. Jingyu, Z. Jun, L. Yuanzhang, S. Zhizhuo and W. Wenming, "Hybrid S-RAID: An Energy-Efficient Data Layout for Sequential Data Storage", Journal of Computer Research and Development, vol. 1, no. $50,(\mathbf{2 0 1 3})$.

[3] Z. Guangyan and Q. Jianping, "An Approach for Migrating Data Adaptively in Hierarchical Storage Systems", Journal of Computer Research and Development, vol. 8, no. 49, (2012).

[4] J. Kim and D. Rotem, "Energy proportionality for disk storage using replication", In Proceedings of the 14th International Conference on Extending Database Technology, vol. 8, no. 72, (2011).

[5] H. Li, "REST, A Redundancy-Based Energy-Efficient Cloud Storage System", Proceedings of the 2012 13th International Conference on Parallel and Distributed Computing, Applications and Technologies. IEEE Computer Society, (2012) December, pp. 537-542, Beijing, China.

[6] S. Long, Y. Zhao and W. Chen, "A three-phase energy-saving strategy for cloud storage systems", Journal of Systems and Software, vol. 1, no. 87, (2014).

[7] S. Q. Long and Y. L. Zhao and W Chen, "MORM, A Multi-objective Optimized Replication Management strategy for cloud storage cluster", Journal of Systems Architecture, vol. 2, no. 60, (2013).

[8] Q. Wei, B. Veeravalli, B. Gong, L. Zeng and D. Feng, "CDRM, A Cost-effective Dynamic Replication Management Scheme for Cloud Storage Cluster", 2010 IEEE International Conference on Cluster Computing, (2010) November, pp. 188-196, New York, USA

[9] Q. Zhu and Y. Zhou, "Power-aware storage cache management. IEEE Transactions on Computers", vol. 5, no. 54, (2005).

[10] P. E. Bianchini, "Energy Conservation Techniques for Disk Array-Based Servers", Proceedings of the 18th International Conference on Supercomputing (ICS), (2004) June, pp. 71-73, New York, USA.

[11] C. Weddle, M. Oldham, Q. Jin, W. A. -I. Andy, R. Peter and G. Kuenning, "PARAID, A Gear-Shifting Power-Aware RAID”, ACM Transactions on Storage, vol. 3, no. 3, (2007).

[12] R. T. Kaushik and, M. Bhandarkar 2010.GreenTDCS: towards an energy-conserving, storage-efficient, hybrid hadoop compute cluster. In Proceedings of the 2010 international conference on Power aware computing and systems, (2010) March 1-9; Berkeley,USA

[13] R. T. Kaushik, L. Cherkasova, R. Campbell and K. Nahrstedt, "Lightning, self-adaptive, energy-conserving, multi-zoned, commodity green cloud storage system", In Proceedings of the 19th ACM International Symposium on High Performance Distributed Computing, HPDC, (2010) June, pp. 332-35, New York, USA.

\section{Authors}

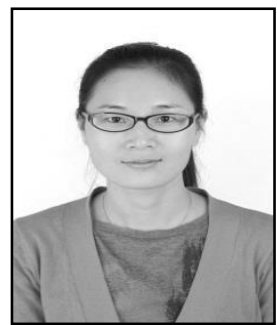

Li Zhouis, she is an associate professor of School of Computer Scienceand Technology, HangzhouDianzi University, China. She is with the Grid and ServiceComputing Lab in HangzhouDianzi University.Before joiningHangzhou Dianzi University, she was a Master candidatein Hangzhou Dianzi University from2003 to 2008. Her current research areas include cloud computing,virtualization,etc

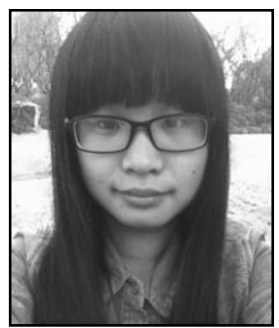

Chi Dong, she is a Master candidate of School of Computer Science and Technology, Hangzhou Dianzi University, China. She is with the Grid and Service Computing Lab in Hangzhou Dianzi University. Before joining Hangzhou Dianzi University, she was a University student in JIAXING University from 2008 to 2012.Her current research areas include distributed Cloud Storage, Energy Management, etc. 
Xindong You, she is a lecturer of School of Computer Science and Technology, Hangzhou Dianzi University, China. She is with the Grid and Service Computing Lab in Hangzhou Dianzi University. Before joining Hangzhou Dianzi University, she was a PhD candidate in Northeastern University from 2002 to 2007. She received her PhD degreein 2007. Her current research areas include distributed computing, Cloud Storage, Energy Management, etc.

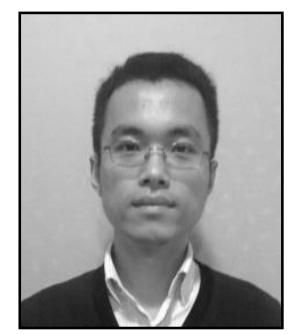

Jie Huang, he is a lecturerof School of Computer Scienceand Technology, HangzhouDianzi University, China. Heis with the Grid and Service Computing Lab in HangzhouDianzi University.Before joiningHangzhou Dianzi University,he was a PhD candidatein Zhejiang University from2003 to 2008 . He received his $\mathrm{PhD}$ degree in 2008. He current research areas include cloud computing,virtualization, geo-computation, etc. 